\title{
Cross talk for angle- and wavelength-multiplexed image plane holograms
}

\author{
Kevin Curtis* and Demetri Psaltis \\ Department of Electrical Engineering, California Institute of Technology, MS 116-81, Pasadena, California 91125 \\ Received April 11, 1994 \\ The cross talk for image plane holograms is calculated and compared with previously derived results for Fourier \\ plane holograms. Image plane storage is found to have significantly smaller cross talk.
}

Previous calculations of the signal-to-noise ratio (SNR) that was due to cross talk between holograms for multiplexing Fourier plane holograms ${ }^{1-3}$ showed that the edges of the recalled image have more noise. This is because Fourier plane holograms map spatial frequency at the hologram into location at the reconstruction plane. For the spacing in angle or wavelength used, ${ }^{1,2}$ the Bragg matching condition only exactly minimizes the cross talk at the center of the image; off-axis pixels have worse SNR than pixels on the optical axis. On the other hand, when image plane holograms are stored, each pixel is reconstructed as an average over all recorded spatial frequencies and as a result we expect each pixel to have the same SNR. We show that this leads to a SNR for image plane holograms that is higher than the worst-case SNR for Fourier plane holograms.

In this Letter we calculate the SNR that is due to cross talk between image plane holograms purely from geometrical constraints. First, the details of the angle-multiplexing case are presented. Then, the calculation for wavelength is summarized because it is similar to the angle-multiplexing case. This Letter contains two main results. First, we show that for image plane holograms the SNR is independent of the pixel location at the output plane. We also show that the worst-case SNR for image plane is better than the worst-case SNR for Fourier-transformed holograms recorded with the same parameters.

Image plane holograms are angle multiplexed in a volume holographic medium with the setup shown in Fig. 1. We have used two $4-F$ systems to image the input onto the material and then to the output plane. The signal at the intermediate plane $\left[\left(x_{1}, y_{1}\right)\right.$ and $\left.\left(x_{2}, y_{2}\right)\right]$ is the Fourier transform of the stored image. Therefore, we can use the previously derived ${ }^{1}$ result for the cross talk of angle-multiplexed Fourier plane holograms to calculate the field at plane $\left(x_{2}, y_{2}\right)$ :

$$
\begin{array}{r}
E\left(x_{2}, y_{2}\right) \approx \sum_{m=-M}^{M-1} F_{m}\left(-\frac{x_{2}}{\lambda F_{3}}-\frac{\Delta K_{m i x}}{2 \pi},-\frac{y_{2}}{\lambda F_{3}}-\frac{\Delta K_{m i y}}{2 \pi}\right) \\
\times \operatorname{sinc}\left\{\frac { t } { 2 \pi } \left[\Delta K_{m i z}+\frac{1}{F}\left(\Delta K_{m i x} x_{2}+\Delta K_{m i y} y_{2}\right)\right.\right. \\
\left.\left.+\frac{\lambda}{4 \pi}\left(\Delta K_{m i x}^{2}+\Delta K_{m i y}^{2}\right)\right]\right\},
\end{array}
$$

where $\Delta \mathbf{K}_{m i}=\mathbf{k}_{m}-\mathbf{k}_{i}$ is the difference between the $m$ th reference wave vector $\mathbf{k}_{m}$ and the illuminating beam's wave vector $\mathbf{k}_{i}$. Also, $\Delta K_{m i \alpha}$ is the component of $\Delta \mathbf{k}_{m i}$ in the $\alpha$ direction and $F_{m}(u, v)$ is the Fourier transform of $f_{m}(x, y)$. The grating vector $\Delta \mathbf{K}_{i j \alpha}$ 's are given by $\Delta K_{i j x}=0, \Delta K_{i j y}=(2 \pi / \lambda f)\left(y_{i}-\right.$ $\left.y_{m}\right) \cos \theta+\left(\pi / \lambda f^{2}\right)\left(y_{m}{ }^{2}-y_{i}{ }^{2}\right) \sin \theta$, and $\Delta K_{i j z}=$ $(2 \pi / \lambda f)\left(y_{i}-y_{m}\right) \sin \theta+\left(\pi^{2} / \lambda f^{2}\right)\left(y_{i}^{2}-y_{m}^{2}\right) \cos \theta$. Also, in relation (1), $t$ is the thickness of the material in the $z$ direction. Note that the above result does not require the imaging to be performed by a $4 F$ system. This was only used in Fig. 1 for clarity of presentation.

Setting $\theta=90^{\circ}$ to maximize the storage capacity, taking the Fourier transform of relation (1), and using the definition of the Fourier transform of $f_{m}$ results in the following expression for the field at the output plane $\left(x_{3}, y_{3}\right)$

$$
\begin{array}{r}
E\left(x_{3}, y_{3}\right) \approx \sum_{m=-M}^{M-1} \iint \mathrm{d} x_{2} \mathrm{dy}_{2}\left(\iint \mathrm{d} x \mathrm{~d} y f_{m}(x, y)\right. \\
\left.\times \exp \left\{-i 2 \pi\left[-\frac{x_{2}}{\lambda F} x+\left(-\frac{y_{2}}{\lambda F}-\frac{\Delta K_{m i}}{2 \pi}\right) y\right]\right\}\right) \\
\times \operatorname{sinc}\left[\frac{t}{\lambda f}\left(y_{i}-y_{m}\right)+\frac{t y_{2}}{2 \lambda f^{3}}\left(y_{m}^{2}-y_{i}^{2}\right)\right] \\
\quad \times \exp \left(-i 2 \pi \frac{x_{3} x_{2}}{\lambda F}\right) \exp \left(-i 2 \pi \frac{y_{3} y_{2}}{\lambda F}\right) .
\end{array}
$$

The above integrals can be evaluated to give us a closed-form expression for the field at the $\left(x_{3}, y_{3}\right)$ plane:

$$
\begin{aligned}
E\left(x_{3}, y_{3}\right) & \approx \sum_{m=-M}^{M-1} \frac{1}{A} \exp \left(-i \frac{2 \pi y_{3} B}{A}\right) \int_{-A / 2-y_{3} / A}^{A / 2-y_{3} / A} \\
& \times \mathrm{d} y f_{m}\left(x_{3}, y\right) \exp \left[i 2 \pi\left(\frac{B}{A}+\frac{\Delta K_{m i}}{2 \pi}\right) y\right],
\end{aligned}
$$

where $A=\left(t / 2 f^{2}\right)\left(y_{m}^{2}-y_{i}^{2}\right)$ and $B=(t / \lambda f)\left(y_{i}-y_{m}\right)$.

To estimate the noise-to-signal ratio (NSR), we divide the total average noise intensity by the magnitude squared of the signal. We assume that each pixel of the stored images is an independent random variable taking the values one and minus one with equal probability. This type of image gives the worst-case SNR and is used for all cases in this Letter. The SNR due to cross talk for images consisting of ones and zeros is approximately two times higher 


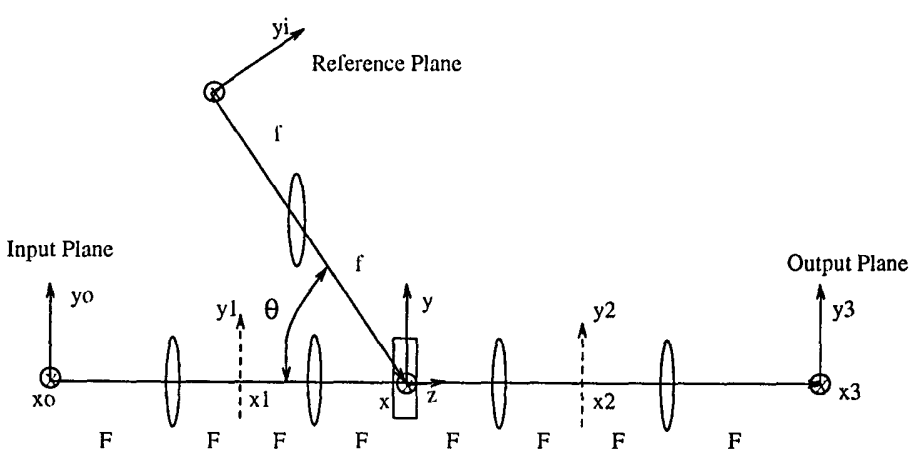

Fig. 1. Recording and readout geometry for angle multiplexing.

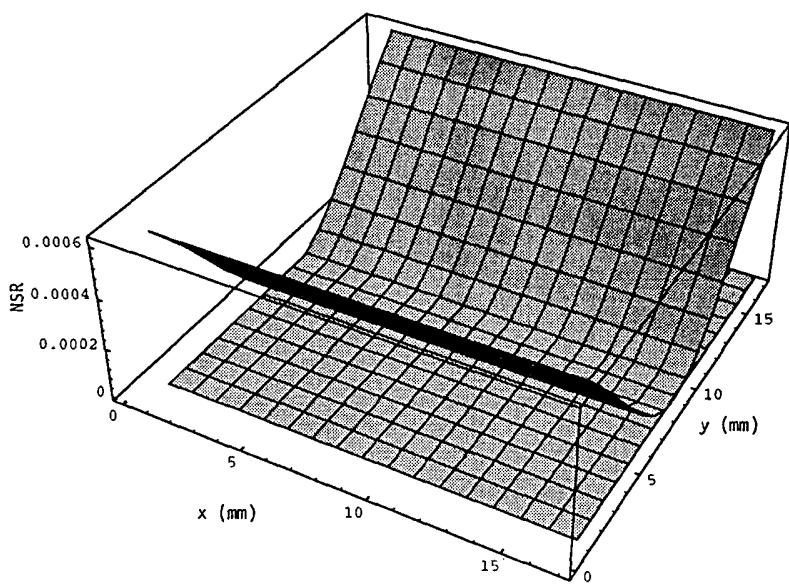

Fig. 2. NSR versus position on the output plane for angle-multiplexed Fourier plane (the valley-shaped curve) and image plane (flat plane) holograms.

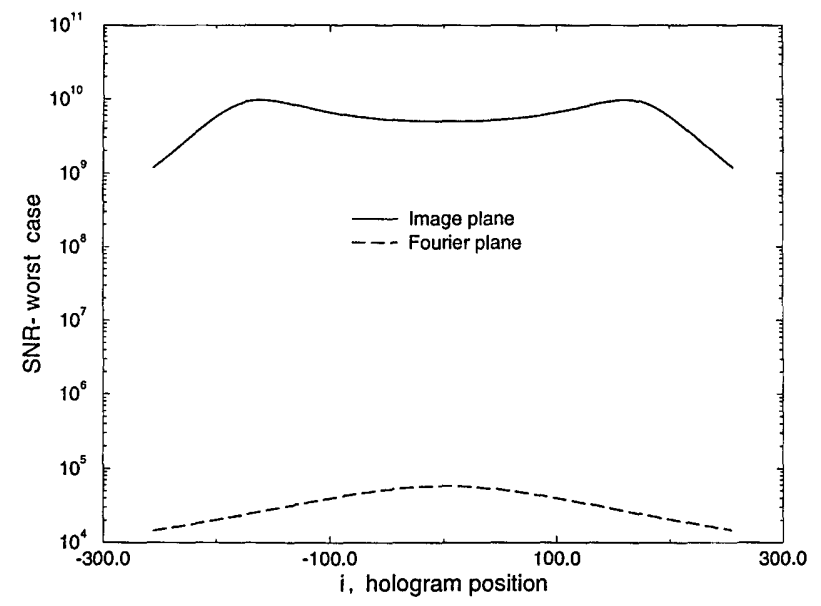

Fig. 3. SNR versus hologram number (i) for angle-multiplexing case.

because only one-half as many gratings are recorded. For images consisting of ones and minus ones, the NSR is given by

$$
\mathrm{NSR}=\sum_{m \neq i} \operatorname{sinc}^{2}\left[\frac{t}{\lambda f}\left(y_{i}-y_{m}\right)+\frac{\pi t}{4 \lambda f^{4}}\left(y_{m}^{2}-y_{i}^{2}\right)^{2}\right] .
$$

To minimize the noise, the reference pixels ( $y_{i}$ 's) need to be spaced by $\lambda f / t$, which is exactly the same spacing as for Fourier plane holograms. Notice that the NSR is independent of pixel position $\left(x_{3}, y_{3}\right)$ at the output plane. Figure 2 shows the NSR versus position at the output plane for $N=2011$ holograms for both image plane and Fourier plane holograms ${ }^{1}$ stored with $F=30 \mathrm{~cm}, t=1 \mathrm{~cm}$, and $\lambda=500 \mathrm{~nm}$. The noise for the image plane case is the flat plane near the bottom, and the Fourier plane case has dependence on $y$.

With the spacing in angle described above, the SNR for the worst pixel at the output plane as a function of hologram angular position in the recording schedule (i) was calculated with $N=513$ and the same parameters given above. The result is shown in Fig. 3. Notice that for both image and Fourier plane holograms the holograms with the worst SNR are furthest from $90^{\circ}(i=M$ or $i=-M)$. Also, for image plane, the holograms stored a little off $90^{\circ}$ have better SNR than the hologram stored at $90^{\circ}$. As more holograms are added the gratings rotate down in grating space with respect to the hologram stored directly at $90^{\circ}$. This rotation increases the noise in the worst spatial frequency but also lowers the average noise across the image. Because image plane cross talk results from some average over all of the spatial frequencies, this

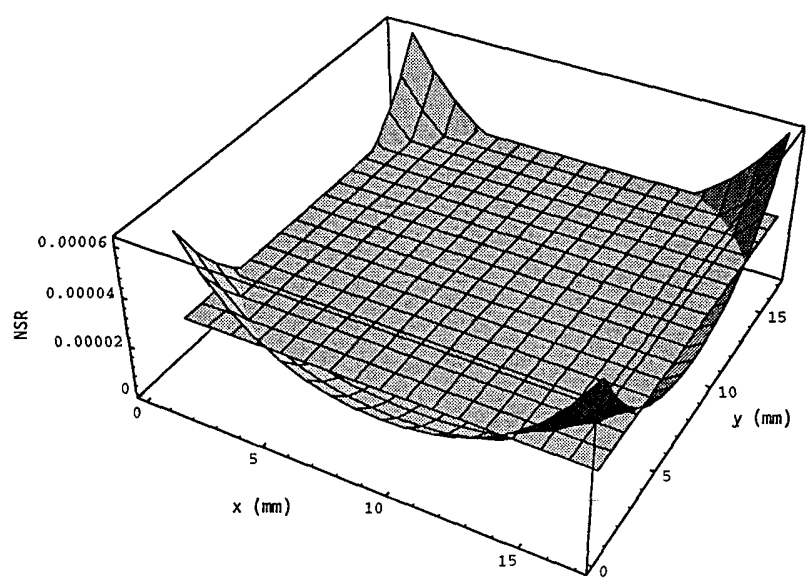

Fig. 4. NSR versus position on the output plane for wavelength-multiplexed Fourier plane (the bowl-shaped curve) and image plane (flat plane) holograms.

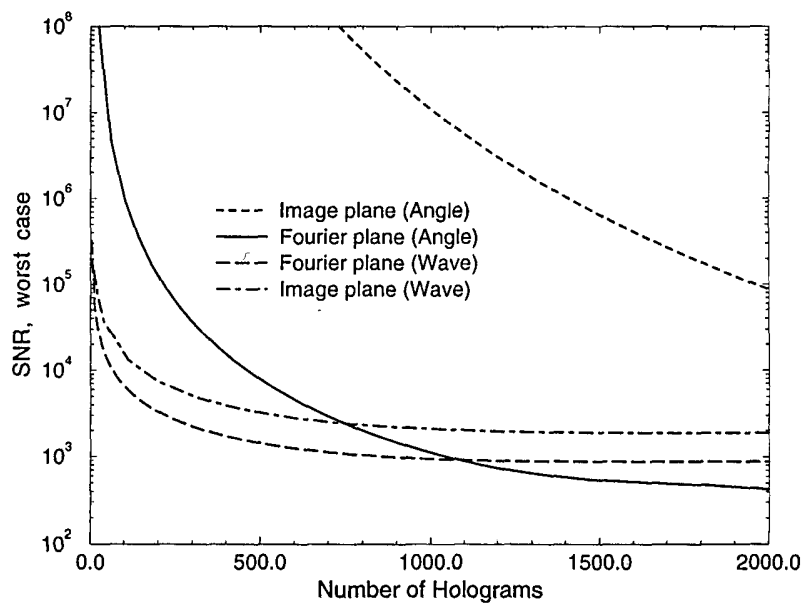

Fig. 5. Worst-case SNR for Fourier plane and image plane holograms versus total number of holograms stored. 
rotation explains why the holograms off of $90^{\circ}$ have slightly better SNR than the hologram stored at $90^{\circ}$.

By analogy with the angle-multiplexing case given above, the diffracted field at the $x_{2}, y_{2}$ plane for pure reflection holograms can be expressed using the results of Ref. 2 as

$$
\begin{aligned}
& E\left(x_{2}, y_{2}\right) \approx \sum_{m=-M}^{M-1} F_{m}\left(-\frac{x_{2}}{\lambda_{i} F},-\frac{y_{2}}{\lambda_{i} F}\right) \\
& \times \operatorname{sinc}\left[-2 t\left(\frac{1}{\lambda_{m}}-\frac{1}{\lambda_{i}}\right)+\frac{t\left(x_{2}^{2}+y_{2}^{2}\right)}{2 F^{2}}\left(\frac{\lambda_{m}}{\lambda_{i}^{2}}-\frac{1}{\lambda_{i}}\right)\right] .
\end{aligned}
$$

Taking the Fourier transform of this expression, multiplying by the complex conjugate, and taking the expected value results in an average intensity at the output plane of

$$
\begin{aligned}
& I_{i}\left(x_{3}, y_{3}\right) \approx \sum_{m=-M}^{M-1} \mid \iint \mathrm{d} w_{x} \mathrm{~d} w_{y} \\
& \times\left.\operatorname{sinc}\left[-2 t\left(\frac{1}{\lambda_{m}}-\frac{1}{\lambda_{i}}\right)+t\left(\lambda_{m}-\lambda_{i}\right)\left(w_{x}^{2}+w_{y}^{2}\right)\right]\right|^{2},
\end{aligned}
$$

where the integrals are over the finite spatial frequencies in the $x_{2}, y_{2}$ plane $\left(w_{x}=x_{2} / \lambda_{i} F, w_{y}=\right.$ $\left.y_{2} / \lambda_{i} F\right)$. Notice that the right-hand side of relation (6) is independent of $x_{3}$ and $y_{3}$ and therefore the noise for image plane wavelength multiplexing is also independent of location on the output plane. Relation (6) can be numerically evaluated and the SNR calculated by taking the result for $m=i$ and dividing it by the result for $m \neq i$.

Figure 4 shows the NSR versus position at the output plane for both image plane and Fourier plane holograms. The curves are for $N=41$ and the same system parameters as given for angle multiplexing. Because the noise for wavelength-multiplexed Fourier plane holograms is a two-dimensional quadratic bowl (rather than a one-dimensional valley), the averaging effect of the image plane format for wavelength multiplexing is not so strong as in the angle-multiplexing case. The image plane result is still independent of location on the output plane and the noise for wavelength-multiplexed image plane holograms is between two and three times less than the worst-case pixel of the Fourier plane hologram.

The SNR versus hologram position in wavelength in the recording schedule has the same dependence as in the Fourier case. ${ }^{2}$ It is flat expect at the edge of the schedule where the holograms have fewer neighbors and therefore have slightly better SNR.

Figure 5 shows the worst-case (in position at the output plane and position in the recording schedule) SNR for angle- and wavelength-multiplexed holograms that are stored in both image plane and Fourier plane formats as a function of the total number of holograms stored. For both multiplexing schemes image plane holograms have higher worstcase SNR than do Fourier plane holograms. The average SNR for angle-multiplexed Fourier holograms is also lower than the worst-case SNR for image plane. In addition, this higher SNR is the same for every pixel in a given hologram, whereas for Fourier-transformed holograms the edges of the image have much lower SNR than the middle of the reconstructed image. ${ }^{1,2}$

We thank the U.S. Air Force Office of Scientific Research, the Advanced Projects Research Agency, and Northrop Corporation for their support and Geoffrey Burr and Ajay Chugh for their helpful discussions.

*Present address, AT\&T Bell Laboratories, Murray Hill, New Jersey 07974.

\section{References}

1. C. Gu, J. Hong, I. McMichael, R. Saxena, and F. H. Mok, J. Opt. Soc. Am. A 9, 1978 (1992).

2. K. Curtis, C. Gu, and D. Psaltis, Opt. Lett. 18, 1001 (1993).

3. A. Yariv, Opt. Lett. 18, 652 (1993). 